Research Paper

\title{
Silencing of Atp6vlcl Prevents Breast Cancer Growth and Bone Metastasis
}

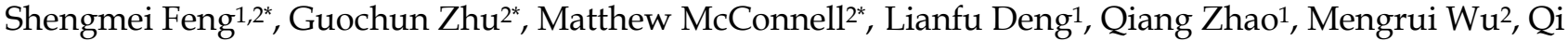 \\ Zhou ${ }^{1}$, Jinshen Wang ${ }^{1}$, Jin Qi ${ }^{1}$, Yi-Ping Li $^{2}$ and Wei Chen ${ }^{2}$ \\ 1. Shanghai Institute of Traumatology and Orthopaedics, Shanghai Key Laboratory for Prevention and Treatment of Bone and Joint Dis- \\ eases with Integrated Chinese-Western Medicine, Ruijin Hospital, Jiao Tong University School of Medicine, Shanghai, P.R.China. \\ 2. Department of Pathology, University of Alabama at Birmingham, Birmingham, Alabama, U.S.A. \\ * Authors contributed equally as first authors.
}

$\triangle$ Corresponding author: Wei Chen, MD, Department of Pathology, University of Alabama at Birmingham, SHEL 810, 1825 University Blvd, Birmingham AL 35294-2182, USA; Tel: 205-975-2607, Fax: 205- 975-4919, E-mail: wechen@uab.edu; and Yi-Ping Li, Ph.D., Department of Pathology, University of Alabama at Birmingham, SHEL 810, 1825 University Blvd, Birmingham AL 35294-2182, USA; Tel: 205-975-2606, Fax: 205-975-4919, E-mail: ypli@uab.edu.

(c) Ivyspring International Publisher. This is an open-access article distributed under the terms of the Creative Commons License (http://creativecommons.org/ licenses/by-nc-nd/3.0/). Reproduction is permitted for personal, noncommercial use, provided that the article is in whole, unmodified, and properly cited.

Received: 2013.02.04; Accepted: 2013.08.07; Published: 2013.09.05

\begin{abstract}
Previous studies have shown that Atp6vlcl, a regulator of the assembly of the $\mathrm{V} 0$ and $\mathrm{VI}$ domains of the V-ATPase complex, is up-regulated in metastatic oral tumors. Despite these studies, the function of Atp6rlcl in tumor growth and metastasis is still unknown. Atp6rlcl's expression in metastatic oral squamous cell carcinoma indicates that Atp6rlcl has an important function in cancer growth and metastasis. We hypothesized that elevated expression of Atp6rlcl is essential to cancer growth and metastasis and that Atp6rlcl promotes cancer growth and metastasis through activation of V-ATPase activity. To test this hypothesis, a Lentivirus-mediated RNAi knockdown approach was used to study the function of Atp6vlcl in mouse 4TI mammary tumor cell proliferation and migration in vitro and cancer growth and metastasis in vivo. Our data revealed that silencing of Atp6 $\mathrm{vlcl}$ in 4TI cancer cells inhibited lysosomal acidification and severely impaired 4TI cell growth, migration, and invasion through Matrigel in vitro. We also show that Atp6rlcl knockdown with Lenti-cls3, a lentivirus targeting Atp6vlcl for shRNA mediated knockdown, can significantly inhibit 4TI xenograft tumor growth, metastasis, and osteolytic lesions in vivo. Our study demonstrates that Atp6rlcl may promote breast cancer growth and bone metastasis through regulation of lysosomal V-ATPase activity, indicating that Atp6rlcl may be a viable target for breast cancer therapy and silencing of Atp6rlcl may be an innovative therapeutic approach for the treatment and prevention of breast cancer growth and metastasis.
\end{abstract}

Key words: Atp6v1c1 (C1), V-ATPase, breast cancer, lysosomal acidification, tumor bone metastasis.

\section{Introduction}

The vacuolar $\mathrm{H}^{+}$-ATPase (V-ATPase) is believed to play an important role in tumor growth and metastasis by increasing $\mathrm{H}^{+}$secretion, allowing tumor cells to survive in hypoxic conditions and the consequent acidic tumor microenvironment $(1 ; 2)$. Furthermore, studies of breast cancer have shown that
V-ATPases located at the plasma membrane of highly metastatic human breast cancer cells are involved in the acquisition of a more metastatic phenotype and that V-ATPase inhibitors decreased the invasion and migration of highly metastatic cells (3). In addition, functional expression of plasmalemmal V-ATPases in 
drug-resistant human breast carcinoma cell lines is also involved in drug-resistance (4). Based on these findings, it is suggested that the V-ATPase complex is a potential target in breast cancer therapy and that it is an excellent candidate for anticancer drugs. However, many current V-ATPase inhibitors (e.g., bafilomycins) are highly toxic and not suitable for clinical use in humans (5). Therefore, defining the exact role and mechanism of V-ATPases in breast cancer cell growth and metastasis is very important, and may reveal a more specific therapeutic target and candidate for novel anticancer drugs.

V-ATPases are composed of an ATP-hydrolytic domain (V1) and a proton-translocation domain (V0), as well as the accessory subunits ac45 and M8-9 (6). The V1 domain is located in the cytoplasm and contains eight different subunits $(\mathrm{A}-\mathrm{H})$. The $\mathrm{V} 0$ domain, an integral membrane bound domain, consists of a, c, $c^{\prime \prime}$, and d subunits in mammals (7-9). The V-ATPase is a tightly coupled enzyme that only exhibits activity when the enzyme is fully assembled with all subunits at the membrane; the $\mathrm{C}$ subunit is primarily responsible for its enzymatic function through the control of a reversible dissociation of the $\mathrm{V} 0$ and $\mathrm{V} 1$ domains (9-12) with Atp6v1c1 acting as a coordinating subunit which mediates V-ATPase assembly by interacting with actin as well as ATP6 subunits v0a, v1g and v1e (7). Atp6v1c1, an isoform of the $C$ subunit, was recently shown to be the most strongly overexpressed gene in oral squamous cell carcinoma in humans compared to other subunits, which suggests that $\mathrm{pH}$ regulation, which can facilitate tumor growth and metastasis, is mainly controlled by Atp6v1c1 (C1) (13). Atp6v1c1 mRNA has also been shown to be expressed in breast cancer cell lines by microarray analysis (Supplementary Material: Fig. S1) (14) (15). However, the exact role and mechanisms of $\mathrm{C} 1$ in tumor growth and metastasis remain unknown. Together, these findings suggest that the subunit $\mathrm{C} 1$ may play an important role in breast cancer cell growth, invasion, and metastatic osteolytic lesions through its role in the regulation of V-ATPases.

To investigate the potential role of ATP6v1c1 in human cancers we used a mouse orthotopic xenograft model using mouse mammary tumor 4T1 (16). Murine ATP6v1c1 (protein: NP_079770.2, mRNA: NM_025494.3) shares considerable homology with the human ATP6v1c1 (protein: NP_001686.1, mRNA: NM_001695.4) when compared using ClustalW2 (17) with a 98 alignment score ( $>98 \%$ amino acid identity) for the proteins, and an 85 alignment score for the mRNA sequences. This extensive homology between the murine and human ATP6v1c1 sequences indicates that they are likely to have similar functions within the cell and cellular milieu, and, further, that a mouse model of ATP6v1c1 knockdown may reasonably be used to recapitulate the human system (18). With that in mind, we found that silencing Atp6v1c1 in metastatic 4T1 mouse mammary cancer cells (4T1) greatly impairs 4T1 cell growth, migration, and invasion in vitro and that Lenti-c1s3 lentivirus can efficiently inhibit breast cancer growth, metastasis and induction of osteolytic lesions in vivo. To our knowledge, this is the first report that $\mathrm{C} 1$ deficiency can block breast cancer growth and metastasis.

\section{Materials and Methods}

Generation of Atp6v1c1-depleted 4T1 cells. 4T1 cells (16) (ATCC) were cultured in RPMI medium 1640 (Gibco/Life Technologies) supplemented with 10\% FBS (Gibco/Life Technologies). 24 hours after plating the lentivirus (Lenti-LacZ or Lenti-c1s3 (19)), generated using the $\mathrm{pLB}$ lentivirus system (Addgene, MA; plasmid \#11619) (20), was added to cells for 8 hours of infection. Loose virus was washed off and media changed at 8 hours and cells were trypsinized 24 hours after infection and resuspended in culture media. Single cell suspensions of the infected cells were seeded in 96-well culture plates. $\mathrm{GFP}^{+}$monoclones which express shRNA were observed as described (19). We chose 4T1-LacZ as a control clone and 4T1-c1s3-1, 4T1-c1s3-2, and 4T1-c1s3-11 clones as c1-depleted 4T1 clones for experiments.

Immunoblotting assays. Immunoblotting was performed as described previously (19). Immunoblotting antibodies were anti-Atp6v1c1 (H-300) and ATP6L (D-12) from Santa Cruz Biotechnology (CA, USA); anti-tubulin (E7) was from the Developmental Studies Hybridoma Bank (IA, USA); Atp6v1a (N1C1) was from Genetex (CA, USA).

Immunohistochemistry assays. Were performed as described (19). Briefly, primary antibody (a-Atp6v1c1 (H-300) (Santa Cruz, CA.), 1:600) was applied overnight at $4^{\circ} \mathrm{C}$. The horseradish peroxidase avidin-biotin complex system (Rabbit Elite ABC Kit; Vector Laboratories) was used to visualize bound antibody. The experiments were performed in triplicate on three independent occasions.

Reverse Transcription-PCR (RT-PCR). These were performed as described (19). The gene-specific primers were: Atp6v1c1 (expected product of $523 \mathrm{bp}$ ) sense 5'-AATAATCTTGCCGTCTCTTCC-3' and antisense 5'-GCGTTTCATACTGCTTAATCC-3', for Atp6v1c2 (common primers for c2-a and c2-b; expected product of 433 bp) sense 5'-AACTTCAAAGTCTAACCTGTCC-3' and antisense 5'-GACAATATCGCTCAGTGTCC-3'; $\beta$-actin (expected product of $517 \mathrm{bp}$ ) sense 5'-CATTGAACATGGCATTGTTACC-3' and antisense 5'-CAGCTCATAGCTCTTCTCCAGG-3'. All 
assays were repeated three times.

Cell growth and migration kinetics assays. Cells were plated in 24 -well plates $\left(1 \times 10^{4} /\right.$ well $)$, after 24,48 , and 72 hours cells were counted from three wells per treatment condition. The experiment was performed on three independent occasions $(n=3)$. Migration was assessed in a wounded monolayer model using the scratch assay, as described (3). Five hours after injury of the monolayer, cell movement was captured under the microscope's medium magnification $(100 \times)$. The experiment was performed in triplicate on three separate occasions $(n=3)$.

Cell invasion assay. Cell invasion was analyzed as described (21) with $10 \%$ FBS as a chemoattractant. After 20 hours of incubation in $37^{\circ} \mathrm{C}, 5 \% \mathrm{CO}_{2}$, cells that had migrated through the membrane where they were fixed with methanol and stained with hematoxylin. Invading cells per field were counted $(n=10)$ in triplicate using a light microscope $(100 \times$ magnification).

Conditioned media assay. 4T1 mammary tumor cells were cultured in 24 -well plates $\left(1 \times 10^{4} /\right.$ well $)$ in standard media conditions (RPMI 1640 supplemented with $10 \%$ FBS) or with $20 \%$ osteoclast conditioned media, from bone marrow derived macrophage osteoclast culture, generated as described (22) for 24 hours after the addition of the conditioned media 4T1 cells were. Experiments were carried out on three separate occasions $(n=3)$.

Primary breast cancer mouse model. All animals were maintained according to the Guide for the Care and Use of Laboratory Animals. For the spontaneous metastasis assay, anesthetized 7-week-old female BALB/c mice were divided into 5 groups with 6 animals per group, and surgically exposed so that PBS or $1 \times 10^{5} 4 \mathrm{~T} 1$ or $c 1$-depleted $4 \mathrm{~T} 1$ cells could be inoculated into the left thoracic (\#2) mammary fat pad in a $50 \mu \mathrm{l}$ volume. During day 10 to day 26 after implantation, we monitored mean tumor diameter (TD) (23) then converted to volume under the assumption that the tumors are roughly spherical. Mice were euthanized after being exposed to X-ray (Faxitron X-ray) on day 28 , and then perfused with $4 \%$ paraformaldehyde in PBS ( $\mathrm{pH}$ 7.4). The primary tumors were surgically removed and weighed. The lungs, liver, femur, and tibia were removed and immersed in $4 \%$ paraformaldehyde in PBS ( $\mathrm{pH} 7.4)$ overnight at $4^{\circ} \mathrm{C}$ before further processing for histological analysis. The femur and tibia were scanned by micro computed tomography (Micro-CT) at $12 \mu \mathrm{m}$ voxel resolution in all three axes on a Scanco Medical $\mu$ CT 40 Micro-CT scanner. The ROI began $0.1 \mathrm{~mm}$ from the lowest point of the growth plate and moved distally for 300 slices. We used the default filters, gauss sigma at 0.7 and support at 1.0. The threshold for bone was $\sim 235 \mathrm{mg}$
$\mathrm{HA} / \mathrm{cm}^{3}$.

In vivo bioluminescent imaging (BLI) of mice. This assay was performed per the protocol from Caliper Life Sciences. The spontaneous metastasis models were generated as described previously then 4T1-LacZ, 4T1-c1s3-1 and 4T1-c1s3-2 cells were infected to express luciferase via a separate lentiviral infection, using Pol-2 promoter driven expression of a chimeric Luc/GFP in a pSico vector (24). Then the cells were injected into the left thoracic (\#2) mammary

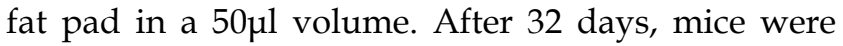
anesthetized by $2.5-3.5 \%$ isofluorane then injected with luciferin (Caliper Life Sciences; $150 \mathrm{mg}$ Luciferin $/ \mathrm{kg}$ body weight) intra-peritoneally (i.p.) 10-15 minutes before imaging using the IVIS ${ }^{\circledR} 100$ bioluminescence imaging system (Xenogen Corp., Alame$\mathrm{da}, \mathrm{CA})$. Images were binned $(2 \times 2)$ with a wide-field of view (D), and f/1.0 with a 300s exposure time.

Hematoxylin and eosin (HE), and tartrate-resistant acid phosphatase (TRAP) staining. These stains were performed as described (25). For metastasis detection, we sectioned regions of the lungs, livers and knee joints from 12 mice per group using $5 \mu \mathrm{m}$ paraffin embedded sections of paraformaldehyde fixed tissues and EDTA decalcified bone, which were stained with hematoxylin and eosin (Azer Scientific, Morgantown, PA). We examined the following regions: bone - femur proximal to the knee; liver - right lobe; and right lung - top/superior lobe, with 20 slides per region per mouse - selected to represent a full sectioning of the region of interest of the organ. Mice for whom metastatic mammary tumor cells were found on any slide of lung, liver, and bone sections were considered positive for metastasis as described (26).

Statistical and data quantification analyses. Experimental data are reported as mean \pm SEM (standard error of measurement) of three independent experiments, or more, as indicated in the respective figure legends and methods. Data were analyzed with the two-tailed Student's $t$-test. $P$-values $<0.05$ were considered significant. Data quantification analyses, including densitometry, were performed using the NIH ImageJ program (27). ${ }^{*}, P<0.05 ; * *, P<0.01$; ***, $P<0.005$ (throughout the paper).

\section{Results}

Single cell clone selection of Atp6v1c1-depleted 4T1 mammary tumor cells. To identify the role of the $\mathrm{C}$ subunit in breast cancer biology, we first checked C1 or C2 expression in 4T1 cells using RT-PCR. We found that $\mathrm{C} 1$ was expressed in $4 \mathrm{~T} 1$ cells while $\mathrm{C} 2$ (C2a and $\mathrm{C} 2 \mathrm{~b})$ was not. We used mouse brain, kidney and lungs as positive controls (Fig. 1A). We selected c1-knockdown single subclones 
of 4T1 cells after infection with lentivirus of Lenti-LacZ or Lenti-c1s3 (19) by viewing $\mathrm{GFP}^{+}$monoclones which express shRNAs. As a control, 4T1-LacZ clone expressing shRNA that specifically targets LacZ. The 4T1-c1s3-1 and 4T1-c1s3-2 clones (representative monoclones of total six monoclones) express shRNA that specifically targets C1 (Fig. 1B). Immunohistochemistry (Fig. 1C) and western blot (Fig. 1D) assays showed that $\mathrm{C} 1$ expression was specifically and significantly reduced in the 4T1-c1s3-1 and 4T1-c1s3-2 clones (reduced by $85 \%$ and $60 \%$ respectively) compared to 4T1-LacZ (Fig. 1D, E). Meanwhile, there was no significant difference in Atp6v1a and Atp6L expression between c1-depleted cells and control cells (Fig. 1D).

Growth, migration, and invasion of Atp6v1c1-depleted 4T1 mammary tumor cells in vitro. We seeded the same number of cells and counted the cultured cells every 24 hours and, after 72 hours, found that the number of 4T1, 4T1-LacZ,
4T1-c1s3-1 and 4T1-c1s3-2 cells were 29.6, 21.8, 6.6 and 15.07 folds (respectively) of that at 0 hour (Fig. 2A). Migration assay revealed that after 5 hours the wound area of both 4T1 and 4T1-LacZ cells had a significant diminution, while the wound area of 4T1-c1s3-1 and 4T1-c1s3-2 cells remained unchanged despite the presence of several individually migrating cells (red arrows) in the 4T1-c1s3-2 cell culture (Fig. 2B). In addition, an in vitro invasion assay showed that the invasion ability through Matrigel of 4T1-c1s3-1 and 4T1-c1s3-2 cells significantly decreased (99\% and 85\% respectively) compared to that of 4T1-LacZ. There was no significant difference between $4 \mathrm{~T} 1$ and 4T1-LacZ cells, but the invasive capacity of 4T1-c1s3-1 cells was significantly less than 4T1-c1s3-2 cells (Fig. $2 \mathrm{C}, \mathrm{D})$. These results indicate that inhibition of $\mathrm{C} 1$ expression greater than $60 \%$ in $4 \mathrm{~T} 1$ cells significantly decreases cell growth, migration, and invasion in vitro.

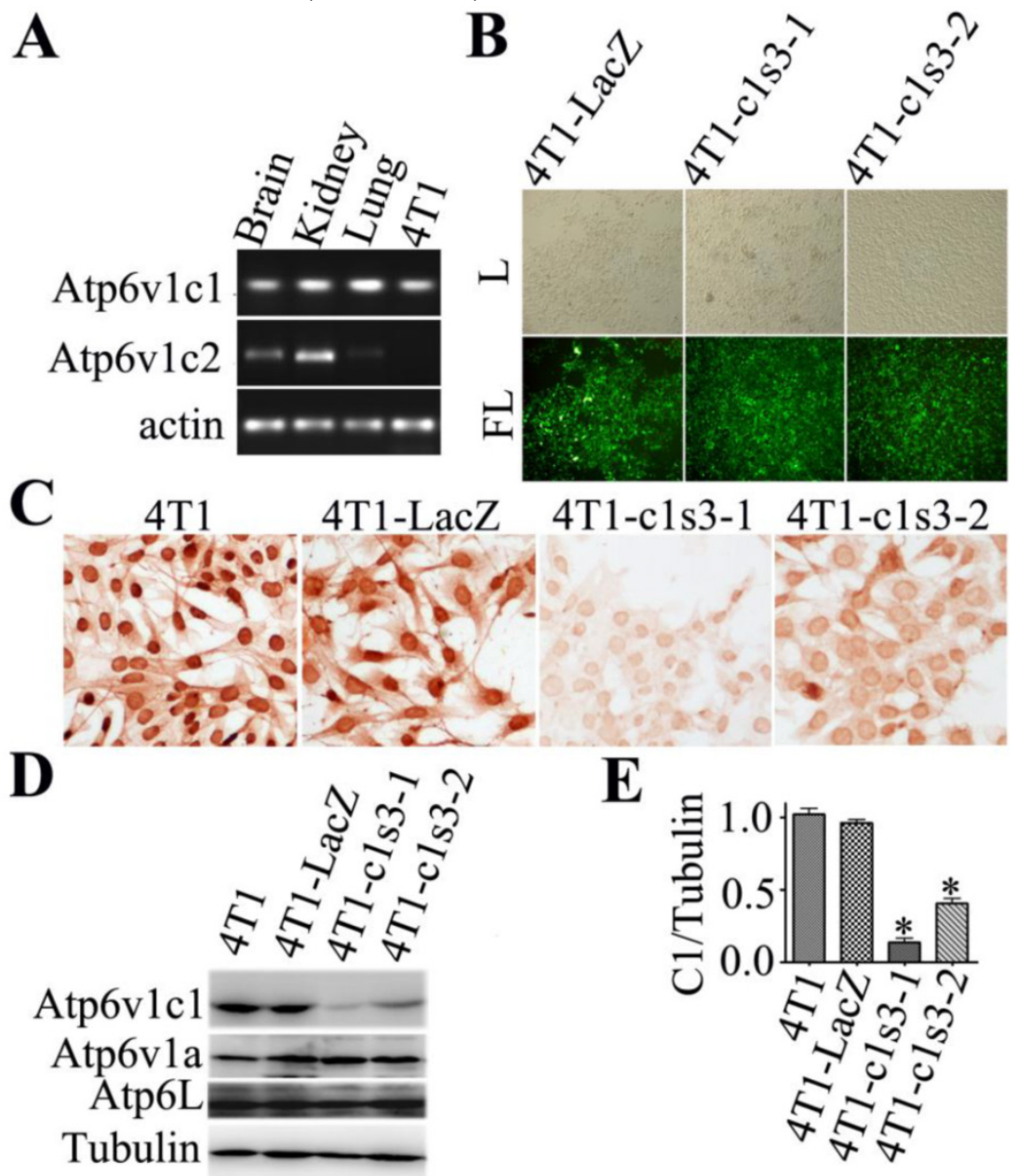

Fig I. Single cell clone selection of Atp6vlcl-depleted 4T I mammary tumor cells. A. RT-PCR assays of three mouse tissues and 4TI cells were performed with gene-specific primers for Atp6vlcl, Atp6vlc2, and $\beta$-actin. B. Select GFP+ single subclones of 4TI cells. The 4TI-LacZ clone expresses siRNA that specifically targets LacZ. The 4TI-cls3-I, 4TI-cls3-2 clones express siRNA that specifically targets $\mathrm{CI}$. Visualized by white light (L) or by fluorescent light (FL) (primary magnification $\times 100$ ). C. Anti-Atp6vlcl immunostaining of different 4TI cells as indicated (primary magnification $\times 100)$. D. Atp6rlcl, Atp6vla and Atp6L expression in different 4TI cells as indicated by Western blotting. E. Quantification of Atp6vlcl protein expression level in different 4TI cells as indicated (normalized to the tubulin level) $(n=3) . * P<0.05$ compared with that of $4 \mathrm{TI}$-LacZ cells. The cells shown were representative of the data $(n=3)$. 

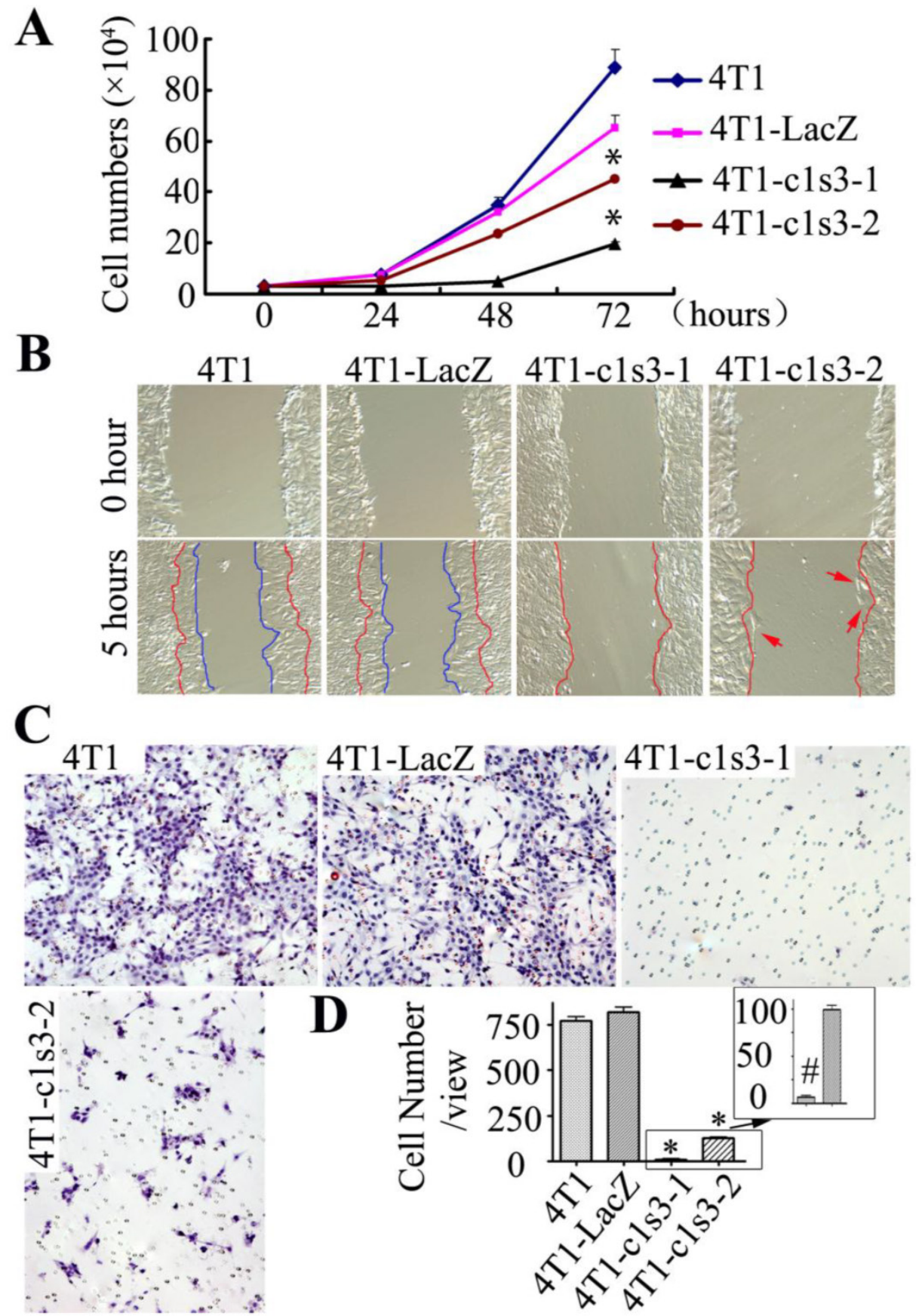

Fig 2. Growth, migration, and invasion of Atp6v I c l-depleted 4TI mammary tumor cells in vitro. A. Cell growth assay of different 4TI cells as indicated. Results are mean \pm s.e.m. $(n=3)$. $* P<0.05$ compared with 4 TI-LacZ by student's t-test at the endpoint. B. Migration assay of different 4 TI cells as indicated. The cells between the red curves and marked by red arrows are the migration cells (primary magnification $\times 100)$. C. Invasion assay of different $4 \mathrm{TI}$ cells as indicated (primary magnification $\times 100)$. D. Quantification of invasion cells per view area. Results are mean \pm s.e.m. $(n=10)$. $* P<0.05$ compared with that of 4 TI-LacZ cells. \# $P<0.05$ compared with that of $4 \mathrm{TI}-\mathrm{cls} 3-2$ cells. The cells shown were representative of the data $(n=3)$.

Atp6v1c1 knockdown inhibits 4T1 mouse mammary tumor growth in vivo. To confirm the results from our in vitro study, we used an orthotopic breast cancer mouse model. We found that the tumors derived from 4T1-c1s3-1 and 4T1-c1s3-2 cells showed significantly slower growth during post-implantation day 0 to day 26, as shown by a smaller tumor volume, and weight 28 days after implantation than those derived from 4T1-LacZ cells. (Fig. 3A, B, C) $(n=24)$. In vivo bioluminescent imaging (BLI) of mice further showed that 4T1-c1s3-1 and 4T1-c1s3-2 mice have lesser tumor volume (day 16) and less detectable metastasis (red arrow indicates metastasis) compared to 4T1-LacZ mice 33 days after inoculation, and there was more necrosis (blue arrow) in the tumors of 4T1-c1s3-2 mice (Fig. 3D, E) ( $n=12)$.

Atp6v1c1 knockdown inhibits primary 4T1 mouse mammary tumor metastasis and prolongs 
survival after xenograft in vivo. To confirm the in vitro result that $\mathrm{C} 1$ knockdown inhibits $4 \mathrm{~T} 1$ cells' migration and invasion, we also analyzed the metastasis in the primary mouse breast cancer model. H\&E staining confirmed that $c 1$-depleted $4 \mathrm{~T} 1$ mice have significantly less metastasis to lungs, liver, and bone compared to 4T1-LacZ mice, which have similar me-

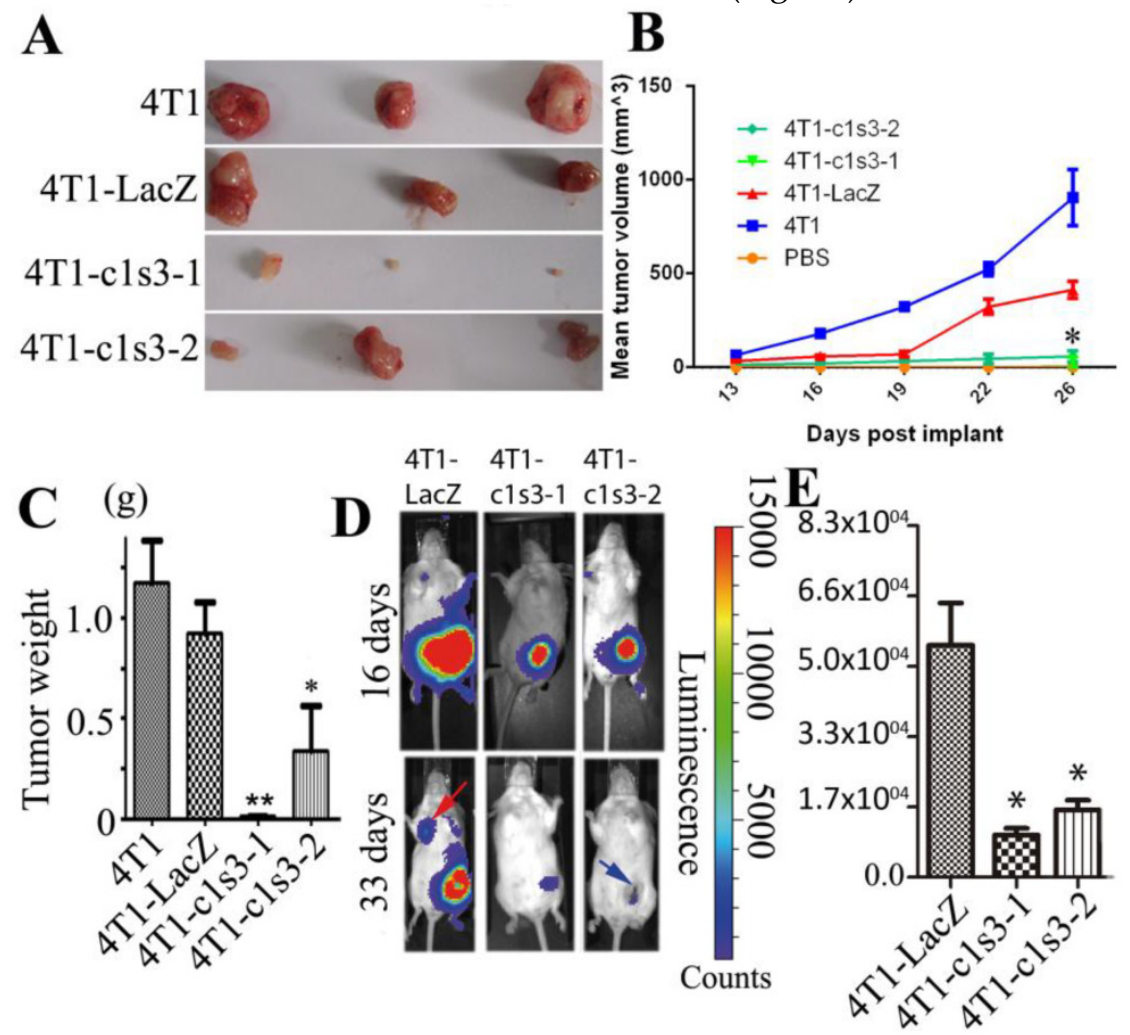

Fig 3. Atp6v l cl knockdown inhibits $4 \mathrm{TI}$ mouse mammary tumor growth and metastasis in vivo. A. Representative tumors from female BALB/c mice 28 days after subcutaneous inoculation in the left thoracic (\#2) mammary gland fat pad with PBS or $1 \times 1054 T 1$ cells infected (or not) as indicated ( $n=24)$. B. The mean tumor diameter (TD) of tumors during the period between post-implantation day 0 and day 26 . Results are mean \pm s.e.m. $(n=24)$. $* P<0.05$ for 4 TI -cl s3-I compared with 4TI-LacZ by student's t-test at the endpoint. C. The weight of tumors on post-implantation day $28(n=24)$. D. Bioluminescent Imaging (BLI) of mice as indicated (Red arrow indicates the metastatic site in BLI of mice and blue arrow indicates tumor necrosis). The images were representative of the data ( $n=12$ ). Counts are photons detected. Images were captured with a $5 \mathrm{~min}$ exposure. E. Whole body flux (photons/sec) quantification of mice injected with different I $\times 1054 \mathrm{TI}$ cells at day 16 as indicated $(n=12)$. Signal measured from a 5 minute exposure. $* P<0.05$ for 4 TI-cls 3 -I compared with 4 TI-LacZ by student's t-test at the endpoint.
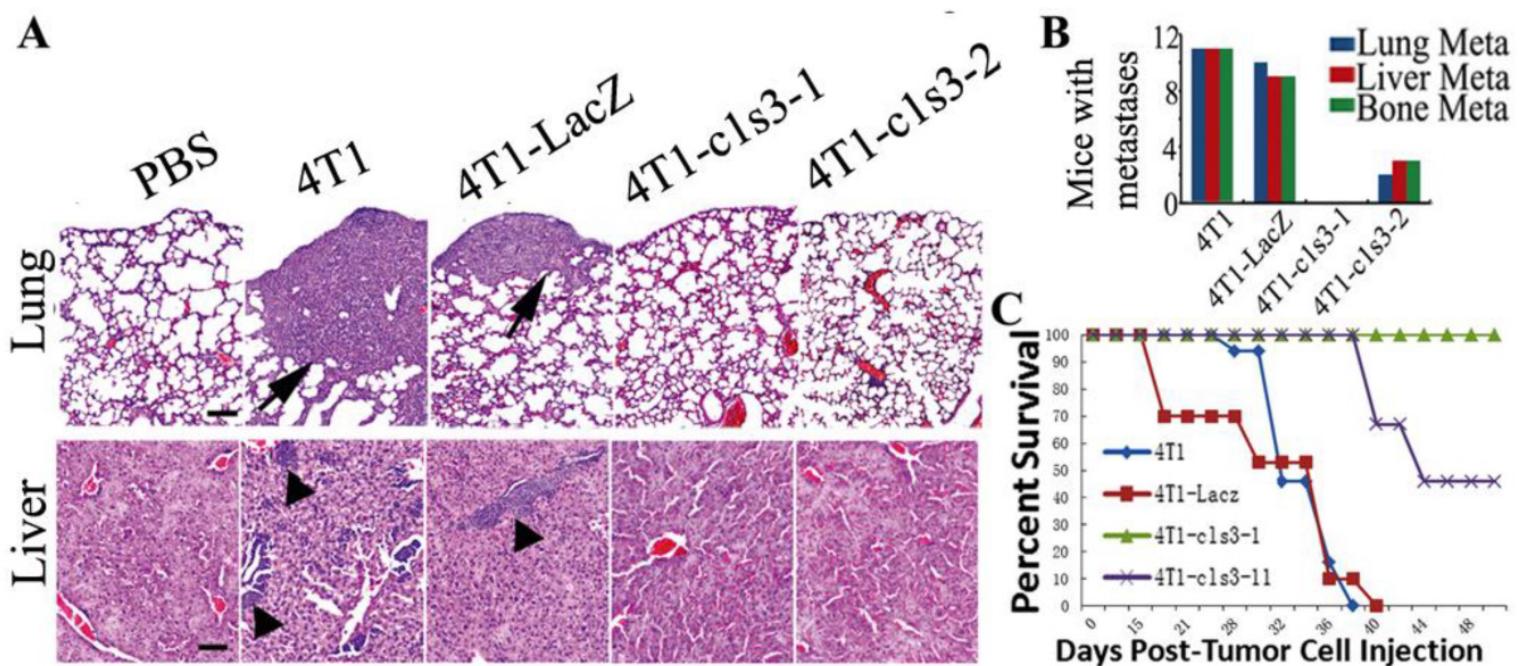

Fig 4. Atp6vlcl knockdown inhibits primary 4TI mouse mammary tumor metastasis and prolongs survival after xenograft in vivo. A. Representative H\&E staining of lungs and livers (scale bar: $100 \mu \mathrm{m}$ ) as indicated. Black arrows indicate the metastases. B. Number of mice bearing metastases in the lungs, liver, and bone from a primary mammary tumor based on H\&E staining at day $33(n=12$ mice). Numbers indicate the number of mice bearing metastases. C. Survival of mice bearing tumors $(n=15)$ over time. 
Fig 5. Bone metastasis and lesions of primary mammary tumors derived from normal and Atp6v/cl-depleted 4TI cells. A. X-ray of representative left femurs and tibias of the primary mammary tumor model $(n=6)$. Red arrows show the bone lesion region. B. Micro-CT images show the region of interest (ROI). Red arrows indicate the bone lesion region. C-G, Results are labeled as I: PBS injected sham control; 2: 4TI xenografted mice; 3: 4TI-LacZ control virus xenografted mice; 4: 4TI-cls3-I ATP6vIcl knockdown xenografted mice; 5:4TI-cls3-2 ATP6rlcl knockdown xenografted mice. C. Quantification of femur relative bone volume over total volume (BV/TV). D. Quantification of femur trabecular bone number (Tb.N). E. Quantification of tibia relative bone volume over total volume (BV/TV). F. Quantification of tibia trabecular bone number (Tb.N). * $p<0.05, * * p<0.0$ I compared to $4 \mathrm{TI}$-LacZ mice. G. H\&E staining of representative left femurs $(n=6)$ (primary magnification $\times 100)$. H. TRAP staining of osteolytic lesions shown in the left femur ROI. Red arrows indicate the TRAP+ osteoclasts. I. Quantification of TRAP+ osteoclasts per view area. Results are mean \pm s.e.m. $(n=5)$ (primary magnification $\times 100$ ). $* P<0.05$ compared with that of $4 \mathrm{TI}-\mathrm{LacZ}$ mice. (GP, growth plate; T.B, trabecular bone; T, Tumor; C.B., cortical bone).

Bone metastasis and lesions arising from murine orthotopic xenografts with normal and Atp6v1c1-depleted 4T1 cells. Most advanced breast cancer patients develop osteolytic bone metastasis and lesions, which are a common cause of morbidity and sometimes mortality, causing extreme pain in advanced cancer patients (28). Therefore, we checked the bones of mice with mammary tumor xenografts. $X$-ray images revealed that the distal end of the left femur of 4T1 or 4T1-LacZ tumor bearing mice has thinner cortical bone and globular intumescences (red arrows), while there was no significant change visible in the bone of the PBS control, 4T1-c1s3-1 and 4T1-c1s3-2 mice (Fig. 5A). The Micro-CT scanning of the region of interest (ROI) in Fig. 5A (Fig. 5B) showed that the femurs and tibias of 4T1 and 4T1-LacZ mice had obvious metastatic osteolytic lesions while those of 4T1-c1s3-1 and PBS groups had no obvious metastatic bone destruction. There was metastatic osteolytic lesion in the femur from 4T1-c1s3-2 mice but no obvious metastatic osteolytic lesion in the tibia. The Micro-CT quantification data (Fig. 5C, D, E, F) and $\mathrm{H} \& \mathrm{E}$ staining further confirmed the results (Fig. 5G). Meanwhile, we found that the number of $\mathrm{TRAP}^{+} \mathrm{OCs}$ on the metaphysis bone surface of 4T1 and 4T1-LacZ mice was significantly greater ( 2 fold) than that of c1-depleted 4T1 mice, while there was no significant difference between 4T1-c1s3-1, 4T1-c1s3-2 and PBS groups (Fig. 5H, I). This data shows that $\mathrm{C} 1$ deficiency can effectively reduce $4 \mathrm{~T} 1$ mammary tumor bone metastasis and metastatic bone lesions which may be attributed to a decrease in OCs or decreased OC activity in local bone metastases.

\section{Discussion}

V-ATPases located at the plasma membrane of highly metastatic human breast cancer cells are involved in the acquisition of a more metastatic pheno-

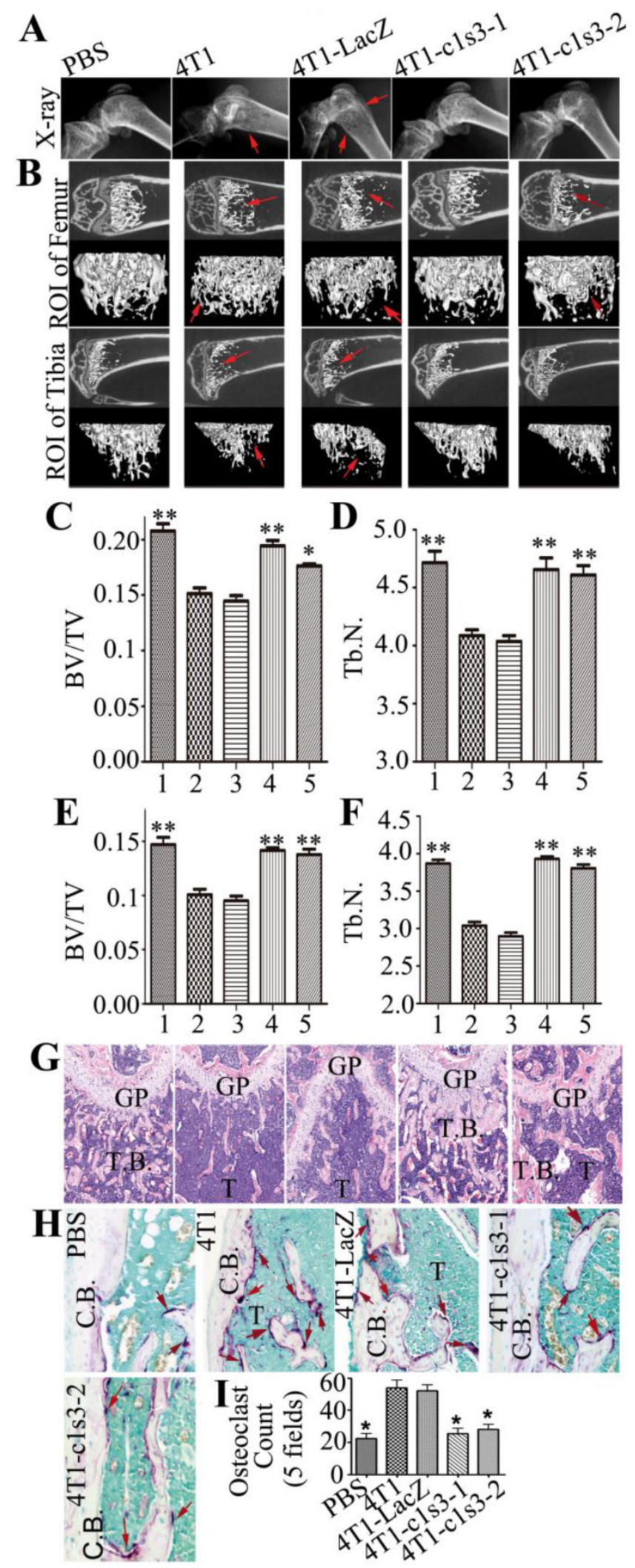

type (3). The C subunit, a component of V-ATPase, regulates V-ATPase enzymatic function through the control of a reversible dissociation of the V0 and V1 domains (9-12). Furthermore, ATP6v1c1 is the most 
strongly overexpressed gene in oral squamous cell carcinoma compared to other subunits of V-ATPase, it was recently suggested to control tumor growth and metastasis (13). Therefore, we hypothesized that Atp6v1c1, as a regulator of V-ATPase activity, plays a key role in breast cancer metastasis.

In this study, we first found that $\mathrm{C} 1$, but not $\mathrm{C} 2 \mathrm{a}$ or $\mathrm{C} 2 \mathrm{~b}$, is expressed in the highly metastatic $4 \mathrm{~T} 1$ mouse mammary cancer cell line as well as human cancer cell lines (Supp. Fig. 1). This is similar to previous findings in which the subunit Atp6v1c1 was ubiquitously expressed in various tissues in mice, but $\mathrm{C} 2 \mathrm{a}$ and $\mathrm{C} 2 \mathrm{~b}$ were expressed specifically in the lungs and kidney respectively $(29 ; 30)$. Therefore, to address the function of $\mathrm{C} 1$ in breast cancer biology, we applied the 4T1 mouse mammary cancer cell line as a model (31) and used lentivirus-mediated siRNA to knockdown $\mathrm{C} 1$ expression in $4 \mathrm{~T} 1$ cells. The Lenti-c1s3 lentivirus that we used here expressed shRNA, which has been previously shown in osteoclasts to specifically and efficiently target Atp6v1c1 (NM_025494.3) but not to target other genes (including subunits ATP6v0a3 and Atp6v1a (19)). Lenti-LacZ lentivirus expressing LacZ shRNA was used as a control. We obtained six $c 1$-depleted $4 \mathrm{~T} 1$ cell clones using GFP as a tool for selection since GFP is co-expressed with the shRNA, and not natively expressed by mice, meaning that $\mathrm{GFP}^{+}$cells express shRNA $(19 ; 20 ; 32 ; 33)$ and chose two of them (4T1-c1s3-1 and 4T1-c1s3-2) as representatives for further studies.

We confirmed 4T1-c1s3-1 and 4T1-c1s3-2 by western blot, immunochemistry and $\mathrm{AO}$ staining. Importantly, C1 knockdown in 4T1 cells significantly inhibits mammary tumor cell growth, migration and invasion in vitro. This result was further supported by our in vivo data that $4 \mathrm{~T} 1$ cells with $\mathrm{C} 1$ knockdown have significantly less tumor volume and less metastasis to the lungs, liver, and bone. These decreases in tumor growth and metastasis were confirmed to further result in an increase in tumor xenograft survival. However, this reduction in tumor metastasis may be a function of the significant reduction in tumor growth seen in the tumors with $\mathrm{C} 1$ knockdown although it is likely that the observed reduction in cancer cell motility and invasiveness is a major factor as well (Fig. 2). In addition, our data is similar to that produced by knockdown of ATP6L expression in hepatocellular carcinoma cells which can effectively retard cancer growth and suppress metastasis (26) and that knockdown of a3 in B16-F10 cells inhibited invasiveness and metastasis to lung and bone (34) which seems to support the hypothesis that extracellular acidification may play a major role in this effect. To our knowledge, this is the first report that $\mathrm{C} 1$ deficiency can block mammary tumor metastasis and growth.
There are many potential causes which may underlie this change in tumor progression and growth in ATP6v1c1-knocked down mice, but previous literature suggests that the reduction in tumor growth and motility may be a function of a constellation of factors including, reductions in the function of low-pH dependent cathepsins, especially in the extracellular milieu, acting in invasion and angiogenesis, presumably to breakdown extracellular matrix (35-37) or via MMP9 which is known to be induced in acidic microenvironments (38-40). Further, the additional growth of cells in the presence of ATP6v1c1 may be a function of the acidic extracellular environment facilitating the release of growth factors such as VEGF and neutralizing cell killing factors such as NOx $(40 ; 41)$ or directly acting on osteoclast precursors to induce invasion and osteoclastogenesis via activation of the NFAT pathway $(42 ; 43)$. Alternatively, inhibition of V-ATPase activity through C1 may result in increased cell death through HIF-1a and increased autophagy (44) for which, perhaps, certain free factors of the V-ATPase may act as signaling vectors. Intriguingly, we have found that, in contrast to control 4T1-LacZ cells, ATP6v1c1-deficient cells are not induced to increase expression of RANKL by treatment with osteoclast conditioned media (Supp. Fig. 2) indicating that knockdown blocks at least one aspect of tumor-osteoclast crosstalk which is known to enhance both tumor growth and osteolysis.

Eighty percent of patients with breast cancer develop bone metastases (45). Bone marrow is a primary site of metastasis, most likely due to its extensive blood supply, available growth factors, and other aspects of the microenvironment (45). Once a tumor lodges in the bone, progressive tumor growth leads to osteoclast-mediated bone loss, which causes debilitating pain and pathological fractures $(45 ; 46)$. In turn, bone resorption by osteoclasts releases growth factors from the bone matrix that stimulate tumor growth and bone destruction (47). This reciprocal interaction between breast cancer cells and the bone microenvironment results in a vicious circle that increases both bone destruction and the tumor burden. Notably, C1 knockdown in $4 \mathrm{~T} 1$ cells effectively reduced primary 4T1 mammary tumor metastatic bone lesions (Fig. 5A-G), which may be due to c1-knockdown $4 \mathrm{~T} 1$ mice having fewer OCs in tumor induced bone lesions compared to $4 \mathrm{~T} 1$ and 4T1-LacZ mice (Fig. 5H, I). This may also be a factor of the decreased tumor growth in the ATP6v1c1 null tumors which could, via a mass effect, impact tumor cytokine production (e.g. Supplementary Material: Fig. S2) and, hence, vicious cycle-related activity (i.e. osteoclastogenesis and bone resorption resulting in tumor growth stimulation) however, we have also shown that knockdown of 
ATP6v1c1 in 4T1 cells results in reduced invasion and migration (Fig. 2B-D) which are necessary for metastasis and which, when diminished, would reduce the efficiency of tumor cell metastasis. Further, acidic microenvironments, such as those induced by tumors and osteoclasts, stimulate osteoclastogenesis through NFAT activity and recruitment of osteoclast precursors $(42 ; 43)$. These results may suggest that high expression of $\mathrm{C} 1$ is involved in 4T1 mammary tumor cell-mediated local immune reaction and inflammation in the bone microenvironment showed by less osteoclasts and osteolytic lesion, and that $\mathrm{C} 1$ deficiency may inhibit the vicious circle between breast-cancer cell growth and cytokine secretion and bone resorption by osteoclasts and decrease breast cancer's metastatic and osteolytic lesions.

The results of our present study show, for the first time, that 4T1 mammary tumor cell V-ATPase-c1 knockdown can efficiently decrease breast cancer growth, metastasis, and formation of osteolytic lesions. Further studies will be required to identify other molecules that might be involved in breast cancer metastasis and metastatic osteolytic lesions through $\mathrm{C} 1$ and confirm their roles. Our results suggest that $\mathrm{C} 1$ would be a useful target for treating breast cancer and even other cancers such as oral squamous cell carcinoma (13) by blocking tumor growth, metastasis and, especially, tumor related osteolysis.

\section{Supplementary Material}

Fig.S1-Fig.S2. http://www.ijbs.com/v09p0853s1.pdf

\section{Abbreviations}

C1: Atp6v1c1; C2: Atp6v1c2; BLI: bioluminescent imaging; HE: hematoxylin and eosin; Micro-CT and $\mu \mathrm{CT}$ : micro computed tomography; OCs: Osteoclasts; RT-PCR: reverse transcription-PCR; ROI: region of interest; TRAP: tartrate resistant acid phosphatase; V-ATPase: vacuolar $\mathrm{H}^{+}$-ATPase.

\section{Acknowledgements}

We thank Ms. Christie Paulson and Mr. Zach Nolen for their excellent assistance with this manuscript. We are grateful for the assistance of Small Animal Bone Phenotyping Core and Histomorphometry and Molecular Analyses Core and Neuroscience Image Core and Neuroscience Molecular Detection Core Laboratory at the University of Alabama at Birmingham (P30 NS0474666). This paper was supported by National Natural Science Foundation of China Grant 30901525, Shanghai Natural Science Foundation Grant 09ZR1428900, Shanghai Education Commission Foundation Grant 10YZ39, and Talents Cultivation Plan of Shanghai Health System XYQ2011050 (all to S.
M. F.). NIH grants AR-055307 (Y.P.L.) and R01-AR-44741(Y.P.L.).

\section{Department where experiments were per- formed}

Department of Pathology, University of Alabama at Birmingham, Birmingham, Alabama, U.S.A. and Shanghai Institute of Traumatology and Orthopaedics, Shanghai Key Laboratory for Prevention and Treatment of Bone and Joint Diseases with Integrated Chinese-Western Medicine, Ruijin Hospital, Jiao Tong University School of Medicine, Shanghai, P.R. China.

\section{Competing Interests}

The authors have declared that no competing interest exists.

\section{References}

1. Martinez-Zaguilan R, Martinez GM, Gomez A, Hendrix MJ, Gillies RJ. Distinct regulation of $\mathrm{pHin}$ and [Ca2+]in in human melanoma cells with different metastatic potential. J.Cell Physiol 1998 Jul;176(1):196-205.

2. Martinez-Zaguilan R, Lynch RM, Martinez GM, Gillies RJ. Vacuolar-type $\mathrm{H}(+)$-ATPases are functionally expressed in plasma membranes of human tumor cells. Am.J.Physiol 1993 Oct;265(4 Pt 1):C1015-C1029.

3. Sennoune SR, Bakunts K, Martinez GM, Chua-Tuan JL, Kebir Y, Attaya MN, Martinez-Zaguilan R. Vacuolar H+-ATPase in human breast cancer cells with distinct metastatic potential: distribution and functional activity. Am.J.Physiol Cell Physiol 2004 Jun;286(6):C1443-C1452.

4. Martinez-Zaguilan R, Raghunand N, Lynch RM, Bellamy W, Martinez GM, Rojas B, Smith D, Dalton WS, Gillies RJ. pH and drug resistance. I. Functional expression of plasmalemmal V-type H+-ATPase in drug-resistant human breast carcinoma cell lines. Biochem.Pharmacol. 1999 May 1;57(9):1037-46.

5. De MA, Iessi E, Logozzi M, Lozupone F, Spada M, Marino ML, Federici C, Perdicchio M, Matarrese P, Lugini L, et al. Proton pump inhibitors induce apoptosis of human B-cell tumors through a caspase-independent mechanism involving reactive oxygen species. Cancer Res. 2007 Jun 1;67(11):5408-17.

6. Xu J, Cheng T, Feng HT, Pavlos NJ, Zheng MH. Structure and function of V-ATPases in osteoclasts: potential therapeutic targets for the treatment of osteolysis. Histol.Histopathol. 2007 Apr;22(4):443-54.

7. Forgac M. Vacuolar ATPases: rotary proton pumps in physiology and pathophysiology. Nat.Rev.Mol.Cell Biol. 2007 Nov;8(11):917-29.

8. Forgac M. Structure and properties of the vacuolar $(\mathrm{H}+)$-ATPases. J.Biol.Chem. 1999 May 7;274(19):12951-4

9. $\mathrm{Xu} \mathrm{T}$, Vasilyeva E, Forgac M. Subunit interactions in the clathrin-coated vesicle vacuolar $(\mathrm{H}(+))$-ATPase complex. J.Biol.Chem. 1999 Oct 8;274(41):28909-15.

10. Parra KJ, Keenan KL, Kane PM. The H subunit (Vma13p) of the yeast V-ATPase inhibits the ATPase activity of cytosolic V1 complexes. J.Biol.Chem. 2000 Jul 14;275(28):21761-7.

11. Kane PM. Disassembly and reassembly of the yeast vacuolar $\mathrm{H}(+)$-ATPase in vivo. J.Biol.Chem. 1995 Jul 14;270(28):17025-32.

12. Sumner JP, Dow JA, Earley FG, Klein U, Jager D, Wieczorek H. Regulation of plasma membrane V-ATPase activity by dissociation of peripheral subunits. J.Biol.Chem. 1995 Mar 10;270(10):5649-53.

13. Otero-Rey EM, Somoza-Martin M, Barros-Angueira F, Garcia-Garcia A. Intracellular $\mathrm{pH}$ regulation in oral squamous cell carcinoma is mediated by increased V-ATPase activity via over-expression of the ATP6V1C1 gene. Oral Oncol. 2008 Feb;44(2):193-9.

14. Wu C, Orozco C, Boyer J, Leglise M, Goodale J, Batalov S, Hodge CL, Haase J, Janes J, Huss JWIII, et al. BioGPS: an extensible and customizable portal for querying and organizing gene annotation resources. Genome Biol. 2009;10(11):R130. PMCID:PMC3091323.

15. Bild AH, Yao G, Chang JT, Wang Q, Potti A, Chasse D, Joshi MB, Harpole D, Lancaster JM, Berchuck A, et al. Oncogenic pathway signatures in human cancers as a guide to targeted therapies. Nature 2006 Jan 19;439(7074):353-7.

16. Lelekakis M, Moseley JM, Martin TJ, Hards D, Williams E, Ho P, Lowen D, Javni J, Miller FR, Slavin J, et al. A novel orthotopic model of breast cancer metastasis to bone. Clin.Exp.Metastasis 1999 Mar;17(2):163-70. 
17. Larkin MA, Blackshields G, Brown NP, Chenna R, McGettigan PA, McWilliam H, Valentin F, Wallace IM, Wilm A, Lopez R, et al. Clustal W and Clustal X version 2.0. Bioinformatics. 2007 Nov 1;23(21):2947-8.

18. Marti-Renom MA, Stuart AC, Fiser A, Sanchez R, Melo F, Sali A. Comparative protein structure modeling of genes and genomes. Annu.Rev.Biophys.Biomol.Struct. 2000;29:291-325.

19. Feng SM, Deng LF, Chen W, Shao JZ, Xu GL, Li YP. Atp6v1c1 is an essential component of the osteoclast proton pump and in F-actin ring formation in osteoclasts. Biochem.J. 2009 Jan 1;417(1):195-203.

20. Kissler S, Stern P, Takahashi K, Hunter K, Peterson LB, Wicker LS. In vivo RNA interference demonstrates a role for Nramp1 in modifying susceptibility to type 1 diabetes. Nat.Genet. 2006 Apr;38(4):479-83.

21. Nam JS, Suchar AM, Kang MJ, Stuelten CH, Tang B, Michalowska AM, Fisher LW, Fedarko NS, Jain A, Pinkas J, et al. Bone sialoprotein mediates the tumor cell-targeted prometastatic activity of transforming growth factor beta in a mouse model of breast cancer. Cancer Res. 2006 Jun 15;66(12):6327-35.

22. Chen W, Zhu G, Hao L, Wu M, Ci H, Li YP. C/EBPalpha regulates osteoclast lineage commitment. Proc.Natl.Acad.Sci.U.S.A 2013 Apr 30;110(18):7294-9. PMCID:PMC3645589.

23. Pulaski BA, Ostrand-Rosenberg S. Animal models for tumor immunology introduction. In: Goligan LI, Bierer B, Margulies DH, et al., editors. Current protocal in immunology. New York: Copyright @2007 by John wiley and sons, Inc.; 1991;4148: 1991.

24. Day CP, Carter J, Bonomi C, Esposito D, Crise B, Ortiz-Conde B, Hollingshead M, Merlino G. Lentivirus-mediated bifunctional cell labeling for in vivo melanoma study. Pigment Cell Melanoma Res. 2009 Jun;22(3):283-95

25. Li YP, Chen W, Liang Y, Li E, Stashenko P. Atp6i-deficient mice exhibit severe osteopetrosis due to loss of osteoclast-mediated extracellular acidification. Nat.Genet. 1999 Dec;23(4):447-51.

26. Lu X, Qin W, Li J, Tan N, Pan D, Zhang H, Xie L, Yao G, Shu H, Yao M, et al. The growth and metastasis of human hepatocellular carcinoma xenografts are inhibited by small interfering RNA targeting to the subunit ATP6L of proton pump. Cancer Res. 2005 Aug 1;65(15):6843-9.

27. Schneider CA, Rasband WS, Eliceiri KW. NIH Image to ImageJ: 25 years of image analysis. Nat.Methods 2012 Jul;9(7):671-5

28. Mundy GR. Metastasis to bone: causes, consequences and therapeutic opportunities. Nat.Rev.Cancer 2002 Aug;2(8):584-93.

29. Sun-Wada GH, Murata Y, Namba M, Yamamoto A, Wada Y, Futai M. Mouse proton pump ATPase C subunit isoforms (C2-a and C2-b) specifically expressed in kidney and lung. J.Biol.Chem. 2003 Nov 7;278(45):44843-51.

30. Sun-Wada GH, Yoshimizu T, Imai-Senga Y, Wada Y, Futai M. Diversity of mouse proton-translocating ATPase: presence of multiple isoforms of the $C$, $d$ and $G$ subunits. Gene 2003 Jan 2;302(1-2):147-53.

31. Tu Q, Zhang J, Fix A, Brewer E, Li YP, Zhang ZY, Chen J. Targeted overexpression of BSP in osteoclasts promotes bone metastasis of breast cancer cells. J.Cell Physiol 2009 Jan;218(1):135-45.

32. Tehrani S, Faccio R, Chandrasekar I, Ross FP, Cooper JA. Cortactin has an essential and specific role in osteoclast actin assembly. Mol.Biol.Cell 2006 Jul;17(7):2882-95.

33. Stewart SA, Dykxhoorn DM, Palliser D, Mizuno H, Yu EY, An DS, Sabatini DM, Chen IS, Hahn WC, Sharp PA, et al. Lentivirus-delivered stable gene silencing by RNAi in primary cells. RNA. 2003 Apr;9(4):493-501

34. Nishisho T, Hata K, Nakanishi M, Morita Y, Sun-Wada GH, Wada Y, Yasui $\mathrm{N}$, Yoneda T. The a3 isoform vacuolar type $\mathrm{H}(+)$-ATPase promotes distant metastasis in the mouse B16 melanoma cells. Mol.Cancer Res. 2011 Jul;9(7):845-55.

35. Joyce JA, Baruch A, Chehade K, Meyer-Morse N, Giraudo E, Tsai FY, Greenbaum DC, Hager JH, Bogyo M, Hanahan D. Cathepsin cysteine proteases are effectors of invasive growth and angiogenesis during multistage tumorigenesis. Cancer Cell 2004 May;5(5):443-53.

36. Gocheva V, Zeng W, Ke D, Klimstra D, Reinheckel T, Peters C, Hanahan D, Joyce JA. Distinct roles for cysteine cathepsin genes in multistage tumorigenesis. Genes Dev. 2006 Mar 1; 20(5):543-56. PMCID:PMC1410800.

37. Joyce JA, Hanahan D. Multiple roles for cysteine cathepsins in cancer. Cell Cycle 2004 Dec;3(12):1516-619.

38. Kato Y, Ozawa S, Tsukuda M, Kubota E, Miyazaki K, St-Pierre Y, Hata R. Acidic extracellular $\mathrm{pH}$ increases calcium influx-triggered phospholipase D activity along with acidic sphingomyelinase activation to induce matrix metalloproteinase- 9 expression in mouse metastatic melanoma. FEBS J. 2007 Jun;274(12):3171-83.

39. Kato $Y$, Lambert CA, Colige AC, Mineur P, Noel A, Frankenne F, Foidart JM, Baba M, Hata R, Miyazaki K, et al. Acidic extracellular $\mathrm{pH}$ induces matrix metalloproteinase- 9 expression in mouse metastatic melanoma cells through the phospholipase D-mitogen-activated protein kinase signaling. J.Biol.Chem. 2005 Mar 25;280(12):10938-44

40. Harhaji L, Popadic D, Miljkovic D, Cvetkovic I, Isakovic A, Trajkovic V. Acidosis affects tumor cell survival through modulation of nitric oxide release. Free Radic.Biol.Med. 2006 Jan 15;40(2):226-35.

41. Taraboletti G, D'Ascenzo S, Giusti I, Marchetti D, Borsotti P, Millimaggi D, Giavazzi R, Pavan A, Dolo V. Bioavailability of VEGF in tumor-shed vesicles depends on vesicle burst induced by acidic $\mathrm{pH}$. Neoplasia. 2006 Feb; 8(2):96-103. PMCID:PMC1578512.

42. Iwai K, Koike M, Ohshima S, Miyatake K, Uchiyama Y, Saeki Y, Ishii M. RGS18 acts as a negative regulator of osteoclastogenesis by modulating the acid-sensing OGR1/NFAT signaling pathway. J.Bone Miner.Res. 2007 Oct;22(10):1612-20.

43. Kim JM, Min SK, Kim H, Kang HK, Jung SY, Lee SH, Choi Y, Roh S, Jeong D, Min BM. Vacuolar-type H+-ATPase-mediated acidosis promotes in vitro osteoclastogenesis via modulation of cell migration. Int.J.Mol.Med. 2007 Mar;19(3):393-400.

44. von SK, Wiedmann RM, Oak P, Schulz S, Zischka H, Wanner G, Efferth T, Trauner D, Vollmar AM. Mode of cell death induction by pharmacological vacuolar H+-ATPase (V-ATPase) inhibition. J.Biol.Chem. 2013 Jan 11; 288(2):1385-96. PMCID:PMC3543021.

45. Cicek M, Iwaniec UT, Goblirsch MJ, Vrabel A, Ruan M, Clohisy DR, Turner RR, Oursler MJ. 2-Methoxyestradiol suppresses osteolytic breast cancer tumor progression in vivo. Cancer Res. 2007 Nov 1;67(21):10106-11.

46. Martin TJ, Moseley JM. Mechanisms in the skeletal complications of breast cancer. Endocr.Relat Cancer 2000 Dec;7(4):271-84.

47. Chirgwin JM, Guise TA. Molecular mechanisms of tumor-bone interactions in osteolytic metastases. Crit Rev.Eukaryot.Gene Expr. 2000;10(2):159-78. 